\title{
Epidemiological Characteristics and Spatiotemporal Patterns of Scrub Typhus in Jiangxi Province, China, 2006-2018
}

\section{Shu Yang}

The Collaboration Unit for Field Epidemiology of State Key Laboratory of Infectious Disease Prevention and Control, Nanchang Center for Disease Control and Prevention. Nanchang 330038, China.

\section{Xiaobo Liu}

State Key Laboratory of Infectious Disease Prevention and Control, National Institute for Communicable Disease Control and Prevention, Chinese Center for Disease Control and Prevention, Beijing 102206

\section{Yuan Gao}

State Key Laboratory of Infectious Disease Prevention and Control, National Institute for Communicable Disease Control and Prevention, Chinese Center for Disease Control and Prevention, Beijing 102206

\section{Baizhou Chen}

School of Geography and Information Engineering, China University of Geosciences. Wuhan 430078, China.

\section{Liang Lu}

State Key Laboratory of Infectious Disease Prevention and Control, National Institute for Communicable Disease Control and Prevention, Chinese Center for Disease Control and Prevention, Beijing 102206

\section{Weiqing Zheng}

The Collaboration Unit for Field Epidemiology of State Key Laboratory of Infectious Disease Prevention and Control, Nanchang Center for Disease Control and Prevention. Nanchang 330038, China.

\section{Renlong Fu}

The Collaboration Unit for Field Epidemiology of State Key Laboratory of Infectious Disease Prevention and Control, Nanchang Center for Disease Control and Prevention. Nanchang 330038, China.

\section{Chenying Yuan}

The Collaboration Unit for Field Epidemiology of State Key Laboratory of Infectious Disease Prevention and Control, Nanchang Center for Disease Control and Prevention. Nanchang 330038, China.

\section{Qiyong Liu}

State Key Laboratory of Infectious Disease Prevention and Control, National Institute for Communicable Disease Control and Prevention, Chinese Center for Disease Control and Prevention, Beijing 102206

\section{Guichang Li}

State Key Laboratory of Infectious Disease Prevention and Control, National Institute for Communicable Disease Control and Prevention, Chinese Center for Disease Control and Prevention, Beijing 102206 Haiying Chen ( $\square$ nccdcchy@126.com ) 
The Collaboration Unit for Field Epidemiology of State Key Laboratory of Infectious Disease Prevention and Control, Nanchang Center for Disease Control and Prevention. Nanchang 330038, China.

\section{Research Article}

Keywords: Scrub typhus, Epidemiological characteristics, Spatiotemporal patterns

Posted Date: December 14th, 2020

DOl: https://doi.org/10.21203/rs.3.rs-118756/v1

License: (c) (i) This work is licensed under a Creative Commons Attribution 4.0 International License. Read Full License 


\section{Abstract}

With the increasing incidence of scrub typhus (ST) in Jiangxi, it is valuable for the control and management to explore the epidemiological characteristics and spatiotemporal patterns of ST. Fisher's exact tests, seasonal decomposition analysis, spatial autocorrelation analysis, and space-time scan statistical analyses were performed to detect the epidemiological features and spatiotemporal patterns of ST. From 2006 to 2018, a total of 5,508 ST cases were reported in Jiangxi, which increased rapidly. Of these, female cases, cases aged 40 years old and above and farmers accounted for $63.42 \%, 88.2 \%$, and $92.2 \%$, respectively. The majority of cases occurred each year between June and October, with a bi-peak seasonal pattern in summer and autumn. The spatiotemporal dynamics of ST varied over the study period with high-risk areas identified in the southern and eastern part of Jiangxi. Seven spatiotemporal clusters were detected using Kulldorff's space-time scan statistic, and the primary cluster covered one county, Nanfeng county. The results of this study demonstrated that targeted intervention should be executed in high-risk counties of ST in Jiangxi to mitigate the growing threat of ST in the province.

\section{Introduction}

Scrub typhus (ST) is a neglected life-threatening vector-borne infectious disease. The disease is transmitted by chigger mites (larval trombiculid mites) infected with the gram-negative intracellular rickettsial bacterium Orientia tsutsugamushi bacteria ${ }^{1-3}$. Human diseases can range from mild (asymptomatic) to lethal, and are generally flu-like (fever, headache, myalgia) in their symptomology 4,5 . Rodents, the primary hosts of mites, are thus indispensable for the survival of chiggers and play a key role in the transmission of $\mathrm{ST}^{3,6-8}$. There are no long-lasting, broadly-protective vaccines available against $\mathrm{ST}$ at present ${ }^{9}$, and early diagnosis and early treatment can significantly reduce the complications and fatality rate.

The majority of ST cases were reported in the "tsutsugamushi triangle" in the Asia-Pacific area including, but not limited to, Korea, Japan, China, India, Indonesia, Thailand, Sri Lanka, and the Philippines ${ }^{10,11}$. It is estimated that one million cases of ST were found each year within this region, and at least one billion people are at risk of becoming infected ${ }^{12,13}$. Moreover, the incidence in all known endemic regions has begun to rise over the last decade ${ }^{14-17}$. Recent evidence from China indicated that ST has expanded to all the provinces across both rural and urban areas, and the incidence of ST has increased in an unprecedented manner, in both historically endemic areas and in new areas not previously identified as having cases ${ }^{18}$. Jiangxi province, a relatively new epidemic focus of ST in southeastern China, has gradually developed into one of the most seriously affected provinces for the disease ${ }^{5}$, with the increase in the number of cases and geographic expansion of $\mathrm{ST}^{18}$. Since the first case of ST reported Shanggao county in $1998^{19}$, the ST epidemic has rapidly spread 48 counties in 2016 according to the national ST surveillance data ${ }^{18}$. However, studies on ST in the area were still limited. 
Techniques of Spatial epidemiological have been widely applied in infectious disease control, prevention and scientific investigations because of the power of quantitative statistics and mapping visualization ${ }^{20-}$ 24. As an infectious disease, the distribution of ST has spatial heterogeneity. To understand the vulnerability of different regions to disease events and the potential geographical location of disease outbreaks is crucial for disease control and prevention. Therefore, this study explored the epidemic characteristics and spatial-temporal patterns of ST in Jiangxi from 2006 to 2018, to provide information for the local health department to implement targeted surveillance and a control policy.

\section{Materials And Methods}

\section{Study area}

Jiangxi province $\left(24^{\circ} 29^{\prime} 14^{\prime \prime}-30^{\circ} 04^{\prime} 44^{\prime \prime} \mathrm{N}, 113^{\circ} 34^{\prime} 36^{\prime \prime}-118^{\circ} 28^{\prime} 58^{\prime \prime} \mathrm{E}\right)$ is situated in central China on the southern bank of the middle and lower reaches of the Yangtze River (Fig. 1A), covering an area of 166, $900 \mathrm{~km}^{2}$, with 11 prefecture cities and 100 counties, and with a population of 44.56 million in 2010 (Fig. 1B). Jiangxi is one of the highest ecosystem service value provinces in China. This province is dominated by mountains and hills, accounting for $78 \%$ of the total land area of the province. This province has a very high forest coverage rate, which was $63.1 \%$ in 2017 ; and the province is ranked second in the forest area behind Fujian province based on statistical data. It has a subtropical monsoon climate, with a mean temperature of $18.9^{\circ} \mathrm{C}$ and average precipitation of $1739 \mathrm{~mm}$ in $2017^{25}$.

Fig 1. The location of the study area. (A) Location of Jiangxi province, in China. (B) Administrative division of the study area. (C) The geographic distribution of five zoographic regions. (Version $10.4 \mathrm{ESRI}$, Redlands, CA, USA, https ://www.esri.com/softw are/arcgi s/arcgi s-for-desktop).

Topographically, the terrain inclines from south to north, and from the outskirts to the central part of Poyang lake. The low elevation area is distributed in central Jiangxi province. Poyang Lake is the largest freshwater lake and one of the top ten ecological function reserves in China, and this province was included in the first batch of National Ecological Civilization Pilot Zones in $2016^{25}$.

As early as in the 1990s, Wang and Song ${ }^{26}$ concluded that Jiangxi might be divided into five zoogeographic regions, including Jiangxi northern bordering on rivers and lakes plain region, Jiangxi central hilly plain region, Jiangxi western mountainous and hilly region, Jiangxi eastern mountainous and hilly region, and Jiangxi southern mountainous region(Fig 1C).

\section{Data source}

ST is a notifiable infectious disease. Every medical institution was required to report to the Chinese Center for Disease Control and Prevention through China's National Statutory Infectious Disease Reporting Information System (CNNDS) (http://www.chinacdc.cn/). Information on ST case reports includes age, occupational, onset and date of diagnosis, case category, and residential address etc. For this study, the daily data from the ST cases in Jiangxi from January 2006 to December 2018 were extracted from 
CNNDS. Demographic data at the county level was obtained from the National Bureau of Statistics of the People's Republic of China (http://www.stats.gov.cn/tjsj/ndsj/). The base map was acquired from the geospatial data cloud (http://www.gscloud.cn/). All cases were geocoded and matched to the corresponding county administrative boundaries using ArcGIS software (Version 10.4, ESRI Inc., Redlands, CA, USA).

ST was diagnosed according to the diagnostic criteria issued by the Ministry of Health of the People's Republic of China (http://www.nhc.gov.cn/wjw/s9491/200802/38814.shtml). For this study, clinically diagnosed and laboratory-confirmed cases were included, and 105 cases were excluded due to invalid addresses (incomplete, incorrect, or outside study area) or suspected cases.

\section{Data analysis}

The epidemiological trends were analyzed using IBM SPSS Statistics for Windows (Version 20.0; IBM Corp., Armonk, NY, USA). A descriptive analysis was utilized to explore the epidemiological characteristics of ST. Fisher's exact tests were used to compare the differences in the age and occupation distributions across different years. A P value of $<0.05$ was considered to be statistically significant, and all of the tests were two-tailed.

A seasonal-trend decomposition of time series analysis was conducted in R software (Version 3.1 AT\&T Bell Laboratories, Auckland, New Zealand). A global spatial autocorrelation analysis and a Local Indicators of Spatial Association (LISA) analysis were implemented in ArcGIS software (Version 10.4, ESRI Inc., Redlands, CA, USA) to analyze the spatial patterns and the potential hotspots associated with the ST incidence at the county level. Global Moran's / Index, which ranged from -1 to 1 , reflects the similarity of attributes in spatial adjacent regions. Moran's / Index $=0$ implied a random spatial distribution. Moran's / Index $<0$ suggested a dispersing spatial distribution, and Moran's / Index $>0$ implied a clustering spatial distribution. Local Moran's / for LISA is a measure of the similarity of difference between the attribute of the observation unit and those of surrounding units, it was calculated to explore significant hot spots (High-High), cold spots (Low-Low), and outliers (High-Low and LowHigh $)^{20}$. A Kulldorff's spatiotemporal scan statistical analysis was used to explore the locations of highrisk spatiotemporal clusters, which can be realized by SaTScan Software (Version 9.4, Martin Kulldorff, National Cancer Institute, Bethesda, MD, USA) ${ }^{27}$. The discrete Poisson probability model by a circular window with a radius was employed for monthly ST from 2006 to 2018 in the counties. The maximum of the spatial and temporal window sizes was defined as $25 \%$ of the at-risk population and $25 \%$ of the period $^{28}$. Likelihood ratio tests and Monte Carlo simulations were used to evaluate the significance of the spatiotemporal clusters $(p<0.05)$.

\section{Ethics approval}

Permission to conduct this study was approved by the Ethics Review Board of the Nanchang Center for Disease Control and Prevention. Ethics approval was not necessary in this study because no work with 
human subjects was directly involved in our study, and all patient records were anonymized and deidentified prior to analysis.

\section{Results}

\section{Epidemiological characteristics}

A total of 5,508 ST cases, including a fatal case, were reported in Jiangxi from 2006 to 2018 . The annual incidence ranged from 0.0092 in 2007 to 2.7200 in 2018 per 100,000 individuals ( $>295$-fold change). Male cases were 2,015 (36.58\%), and female cases were 3493 (63.42\%). During the whole study period, the annual male-to-female ratios were separately $1.25: 1,3: 1,0.69: 1,0.8: 1,0.62: 1,0.77: 1,0.57: 1,0.48: 1$, $0.57: 1,0.59: 1,0.51: 1,0.50: 1,0.68: 1$ (Fig 2).

During the period between 2006 and 2018, the median age of cases was 55 years old, ranged from 4 months to 94 years old. The median age increased from 44 years old (IQRs= 36.5-51 years old) in 2006 to 55 years old (IQRs= 48-64 years old) in 2018. The median age of the ST cases all kept at a higher level across the whole study period (Fig 3). Overall, the ST cases aged 40 years old and above were the most common (88.2\%); the highest proportion was in the age group aged 60 years old and above (36.1\%), 50 59 years old $(31.1 \%)$, and $40-49$ years old $(20.9 \%)$. Additionally, the proportion of older age groups is increasing every year from 2006 to 2018, and the proportion of individuals aged over 60 years old rose from $0.22 \%$ in 2006 to $40.27 \%$ in 2018 . The different age group ratios of the different years were significantly different $\left(\chi^{2}=227.8, p=0.000\right)$ (Fig 4).

The occupation distribution of the ST cases was diverse, including farmers, students, children, workers, and retirees. Most of the ST cases were made up of farmers, followed by retirees, students, and housework or unemployed. Most ST cases were farmers (92.2\%), followed by retirees and students $(1.7 \%$ and $1.6 \%$, respectively). Different occupational ratios of different years existed significant differences $\left(\chi^{2}\right.$ $=283.6, p=0.000)($ (S1 Table).

\section{Seasonal pattern}

A seasonal-trend decomposition of time series analysis was selected as a filtering procedure designed for decomposing a time series into trend, seasonal, and remainder components to explore the various characteristics of periodicity and seasonality of ST. There was a rhythmic vibration in the raw data from 2006 to 2018, and most ST cases occurred yearly between June and October. A bi-peak seasonal pattern was noted, including a major peak in Autumn (between September and October), and a secondary peak in Summer (between June and August). Moreover, there was a rapid increase in the number of reported ST cases during the study period and the variation characteristic of periodicity with the amplitude and the magnitude of the periodical variation increasing (Fig 5).

\section{Spatiotemporal distribution}


The cumulative number of ST affected areas in Jiangxi included 9 prefecture cities and 60 counties during the period between 2006 and 2018. The number of affected prefecture cities ranged from 2 to 9 , and the number of affected counties ranged from 3 to 60 . The numbers of the affected prefecture cities and affected counties showed rapid trends of growing larger (Fig 6). The top five counties with the highest ST incidence rates included Nanfeng county (424.75/100000) of Fuzhou city, and Xunwu county (189.1/100000), Anyuan county (123.55/100000), Longnan county (122.21/100000), and Xinfeng county (77.25/100000) of Ganzhou city, all of which located in Jiangxi eastern mountainous hilly region or Jiangxi southern mountainous region (Fig 7).

\section{Spatial autocorrelation analysis}

In the autocorrelation analysis, from 2007 to 2018, the $P$ values of the Global Moran's / were all less than 0.05, while it was more than 0.05 in 2006 (S2 Table). The global autocorrelation indicated that the spatial distribution of the ST cases was not random except in 2006, but a spatial aggregation distribution. The hotspots (High-High) and outliers of ST transmission in Jiangxi were identified through LISA analysis. High-High hotspots were mainly distributed in southeastern Jiangxi, including Ganzhou city and its neighboring Fuzhou city, however, variations of the location were observed. High-High hotspots were firstly identified in Nankang, Xinfeng, and Longnan counties in 2007, then expanded to surrounding counties and covered the greatest number of counties in 2012, including thirteen counties. Finally, during the entire study period, seven counties of Ganzhou city, including Nankang, Ganxian, Xinfeng, Longnan, Anyuan, Xunwu, and Huichang county, developed into relatively stable High-High hotspots. Furthermore, High-High hotspots also extended to Fuzhou city, from south to east of Jiangxi, three counties, including Nanfeng, Yihuang, and Lichuan, became hotspots successively, and both of Nanfeng and Lichuan county were identified as relatively stable hotspots between 2006 to 2018. It is worth noting that Yifeng and Nanfeng county was High-low outliers in 2006, then Nanfeng county transformed into a High-High hotspot in 2011, 2016, 2017, and 2018. Additionally, the Low-High outliers were also observed in 2008, including Xinfeng, Zhanggong, and Shangyou county (Fig 8).

\section{Spatiotemporal Clusters Analysis}

The distribution of annual average ST incidence and the location of spatial clusters identified by using Kulldorff's spatiotemporal scan statistics for each year from 2006 to 2018 were shown in Fig 5.

Apparently, both the number of counties with increased ST incidence expanded persistently from 2006 to 2014, which caused the formation of a large, contiguous geographic area of ST incidence in southeastern Jiangxi. The primary cluster of ST cases was initially located in Yifeng county of Yichun city, after which the area expanded from 2008 to 2018 and shifted to the southeast, where the primary cluster was identified in southeastern Jiangxi that included Ganzhou city and Nanfeng county of Fuzhou city. Secondary clusters of ST cases were also detected in southeastern Jiangxi, with two to five clusters identified each year (Fig 9).

Additionally, spatiotemporal clusters across the whole study period from 2006 to 2018 were identified by using Kulldorff's spatiotemporal scan statistic. The primary cluster only included a county, Nanfeng 
county of Fuzhou city, which is in the Jiangxi eastern mountainous hilly region. A total of 287,932 human beings were included with a time frame from September 2015 to November 2016. The expected case number was 3.45 , while the observed case number was 567 . The relative risk for the analysis was 183.10 ( $L L R=2359.17, P<0.05)$. It is worth noting that the primary cluster accounted for only $0.65 \%$ of the total population, while included $10.3 \%$ of the total cases during that time. Besides, one $1^{\text {st }}$ Secondary cluster was detected in southern Jiangxi, covering six counties in Ganzhou city. Five $2^{\text {nd }}$ Secondary clusters were identified in southeastern Jiangxi, including eleven counties in Ganzhou city, six counties in Fuzhou city, one county in Yingtan city attributing to eastern Jiangxi, and even one county in Ji'an city attributing to central Jiangxi (Fig 10).

\section{Discussion}

In recent years, the rapid spreading of ST poses a serious public health challenge in Jiangxi. Similar to the whole of China ${ }^{6,29-32}$, the incidence in Jiangxi also exponentially increased between 2006 and 2018. This study, a longitudinal surveillance dataset spanning 13 years in Jiangxi, revealed that the average annual incidence of ST in 2018 was more 295-fold than that in 2006, and the amplitude and the magnitude of ST outbreaks in Jiangxi remained rapid increase during the entire study period. Two factors may have contributed to this result. First, as a natural-focus disease, ST had been paid too little attention where it was previously unknown ${ }^{33}$. Following the discovery of the first confirmed cases of ST in Jiangxi in 1998, enhanced awareness of ST directly resulted in increased surveillance, coupled with the

improvement of the diagnostic tests, and resulted in more cases being diagnosed and reported ${ }^{34}$. Second, it's likely that rodents bearing infected mites may expand their ranges as climate changes, globalization, and urbanization ${ }^{35-37}$. For example, frequent transportation of farmed goods may lead to the migration of rodents carrying infected mites, which might cause the expansion of primal endemic areas and the emergence of new endemic areas ${ }^{38}$. Such expansion may explain the increase of ST incidence in Jiangxi.

The global model showed that the distribution of ST cases in Jiangxi had spatial aggregation. LISA model and spatiotemporal scan analysis indicated that the counties at the highest risks were primarily located in southeastern Jiangxi, including Fuzhou and Ganzhou city. Notably, rodents played a key role in the transmission of ST, and an investigation of rodent host distribution of ST in 1994 in Jiangxi indicated that it existed a significant difference between the dominant rodent species in the south and north Jiangxi ${ }^{26}$. Regrettably, we lack animal host surveillance data until now. Perhaps investigations of the vector host and its natural cycle in Jiangxi will contribute usefully to our knowledge and our ability to prevent ST outbreaks in the future. In addition, by analyzing annual spatiotemporal clustering, the transmission dynamics appeared to shift between south and east Jiangxi. Notably, High-High spots were mainly located in southeastern Jiangxi. The Low-High outliers that were located in this region in 2008, suggested that ST incidence in this region were concentrated in a few counties, which could indicate that the natural foci of ST were still forming in this region as they expanded into southeastern Jiangxi. The High-Low outliers that were located in Yifeng county of Yichun city and Nanfeng county in 2006, suggested that there was only a sporadic outbreak of ST that occurred in Jiangxi around 2006. 
Additionally, Nanfeng county developed into a High-High spot after 2011, which suggested that the natural foci of ST had formed in this region, and the epidemic of ST in Jiangxi could expand from southern Jiangxi to eastern Jiangxi. Therefore, our findings identified established foci in southern Jiangxi and in documenting new foci emerging in the east, where there is an urgent need to enhancing the awareness of local clinicians in diagnosis and treatment, alternative diagnostic methods for ST, and implement targeted intervention of rodent control and habitat modification, public education, and strengthening personal protection.

Our findings showed that the majority of ST cases occurred yearly between June and October, with a bipeak seasonal pattern in summer peaked in August and autumn peaked in October, respectively, which was highly consistent with the planting and picking seasons of "Nanfeng' tangerine" and "Gannan navel orange". Coincidentally, the counties with the highest risk were the main plant areas of "Nanfeng' tangerine" or "Gannan navel orange", with large planting areas and high annual output. Generally, the season for deworming of "Nanfeng' tangerine" trees are from June to August, and the time for picking the first batch of "Nanfeng' tangerine" is from September to October ${ }^{39}$. It's likely that planting and picking of "Nanfeng' tangerine" increased the risk of exposure to ST. Additionally, a case-control study in southern Jiangxi also indicated that Gannan navel orange orchard was the most important site of exposure to $\mathrm{ST}^{40}$. Therefore, local growers should strengthen personal protective measures, including wearing longsleeved shirts and long pants, tightening the neckline and cuffs, and taking a bath in time after work.

Consistent with several studies ${ }^{6,31,32}$, our findings showed that women were more susceptible to ST infections than men, farmers who lived in rural areas accounted for the vast majority, and most cases of ST occurred in the middle-aged and elderly (individuals aged at least 40 years old). A potential explanation was that Jiangxi is known as a "great labor-exporter" 41 , most young and middle-aged males in rural areas were working in other provinces and cities ${ }^{42}$. The result was "geriatrification of agriculture" and "feminization of agriculture", with older and female family members amplifying opportunities for acquiring the disease ${ }^{43}$. Hence, the female farmers aged over 40 years old should be regarded as the key population for ST prevention and control, and personal protection awareness should be strengthened by health education.

Two limitations should be considered when interpreting the findings of this study. Firstly, the bias could exist in this study because all the data used were extracted from a passive surveillance system. Therefore, data quality may be influenced by the availability of diagnostic technology, underreporting. However, the data used in this study were the most comprehensive and reliable data on ST available at national and subnational levels in China. Secondly, the present reporting system was the lack of data on the accurate distribution of mite species, causing it impossible to explore the association among human cases, pathogens, hosts, and vectors.

In conclusion, this is the first systematic report on the epidemiological and spatiotemporal characteristics of ST in Jiangxi. this study illustrated that ST has been spreading widely and increasing rapidly in Jiangxi and that the female farmers aged at least 40 years old were the most susceptible population. The 
majority of ST cases occurred yearly between June and October, with a bi-peak seasonal pattern in summer and autumn. The high-risk areas of the disease were mainly located in Jiangxi southern Jiangxi, spreading to eastern Jiangxi as time advanced. These results showed that ST has become a potential great public health threat to the high-risk population in the affected areas. Further in-depth surveys should be conducted to comprehensively define the epidemiological characteristics of ST. Immediate measures should be taken in the high-risk areas to increase health education and awareness of ST, enhance treatment and diagnostic practices, and persistently improve surveillance of this emerging infectious disease.

\section{Declarations}

\section{Acknowledgments}

This study was supported by the Science and Technology Bureau of Nanchang City (CN) (2020KJZCHTS). The funders had no role in study design, data collection, and analysis, decision to publish, or preparation of the manuscript. We thank all the doctors and staff who have collected and reported ST cases.

\section{Author contributions}

All authors have approved the final version of the manuscript, and have significant contributions to the work. Haiying Chen and Guichang Li initiated the study. Shu Yang collected the data, cleaned the data, performed the analysis and drafted the manuscript. The others revised the manuscript.

\section{Competing interests}

The authors declare no competing interests.

\section{Additional information}

Correspondence and requests for materials should be addressed to Guichang Li or Haiying Chen.

\section{Data availability}

The dataset analyzed is available from the corresponding author for reasonable requests.

\section{References}

1. Bonell, A., Lubell, Y., Newton, P. N., Crump, J. A. \& Paris, D. H. Estimating the burden of scrub typhus: A systematic review. PLoS neglected tropical diseases 11, e0005838, doi:10.1371/journal.pntd.0005838 (2017).

2. Zhang, M. et al. Mixed scrub typhus genotype, Shandong, China, 2011. Emerging infectious diseases 20, 484-485, doi:10.3201/eid2003.121349 (2014). 
3. Elliott, I. et al. Scrub typhus ecology: a systematic review of Orientia in vectors and hosts. Parasit Vectors 12, 513, doi:10.1186/s13071-019-3751-x (2019).

4. Liu, Y. X. et al. Clinical characteristics of the autumn-winter type scrub typhus cases in south of Shandong province, northern China. BMC infectious diseases 9, 82, doi:10.1186/1471-2334-9-82 (2009).

5. Xin, H. L. et al. Evaluation of scrub typhus diagnosis in China: analysis of nationwide surveillance data from 2006 to 2016. Infect Dis Poverty 8, 59, doi:10.1186/s40249-019-0566-0 (2019).

6. Ren, J. et al. Re-emergence of scrub typhus in Zhejiang Province, southern China: A 45-year population-based surveillance study. Travel Med Infect Dis, doi:10.1016/j.tmaid.2019.05.013 (2019).

7. Kuo, C. C., Huang, J. L., Ko, C. Y., Lee, P. F. \& Wang, H. C. Spatial analysis of scrub typhus infection and its association with environmental and socioeconomic factors in Taiwan. Acta Trop 120, 52-58, doi:10.1016/j.actatropica.2011.05.018 (2011).

8. Kuo, C. C., Lee, P. L., Chen, C. H. \& Wang, H. C. Surveillance of potential hosts and vectors of scrub typhus in Taiwan. Parasit Vectors 8, 611, doi:10.1186/s13071-015-1221-7 (2015).

9. Chattopadhyay, S. \& Richards, A. L. Scrub typhus vaccines: past history and recent developments. Hum Vaccin 3, 73-80, doi:10.4161/hv.3.3.4009 (2007).

10. Derne, B., Weinstein, P., Musso, D. \& Lau, C. Distribution of rickettsioses in Oceania: past patterns and implications for the future. Acta Trop 143, 121-133, doi:10.1016/j.actatropica.2014.10.012 (2015).

11. Xu, G., Walker, D. H., Jupiter, D., Melby, P. C. \& Arcari, C. M. A review of the global epidemiology of scrub typhus. PLoS neglected tropical diseases 11, e0006062, doi:10.1371/journal.pntd.0006062 (2017).

12. Kelly, D. J., Fuerst, P. A., Ching, W. M. \& Richards, A. L. Scrub typhus: the geographic distribution of phenotypic and genotypic variants of Orientia tsutsugamushi. Clinical infectious diseases: an official publication of the Infectious Diseases Society of America 48 Suppl 3, S203-230, doi:10.1086/596576 (2009).

13. Prakash, J. A. J. Scrub typhus: risks, diagnostic issues, and management challenges. Res Rep Trop Med 8, 73-83, doi:10.2147/rrtm.S105602 (2017).

14. Ranjan, J. \& Prakash, J. A. J. Scrub typhus re-emergence in India: Contributing factors and way forward. Med Hypotheses 115, 61-64, doi:10.1016/j.mehy.2018.03.019 (2018).

15. Weitzel, T. et al. Endemic Scrub Typhus in South America. N Engl J Med 375, 954-961, doi:10.1056/NEJMoa1603657 (2016).

16. Pradeepan, J. A., Ketheesan, N. \& Murugananthan, K. Emerging scrub typhus infection in the northern region of Sri Lanka. BMC Res Notes 7, 719, doi:10.1186/1756-0500-7-719 (2014).

17. Lee, H. W. et al. Current situation of scrub typhus in South Korea from 2001-2013. Parasit Vectors 8 , 238, doi:10.1186/s13071-015-0858-6 (2015).

18. Li, Z. et al. Epidemiologic Changes of Scrub Typhus in China, 1952-2016. Emerging infectious diseases 26, 1091-1101, doi:10.3201/eid2606.191168 (2020). 
19. R.G.; L. et al. The first report of Tsutsugamushi disease in Jiangxi Province. Chinese Journal of Zoonoses 15, 0-0 (1999).

20. Yang, S. et al. Spatiotemporal Distribution of Tuberculosis during Urbanization in the New Urban Area of Nanchang City, China, 2010-2018. Int J Environ Res Public Health 16, doi:10.3390/ijerph16224395 (2019).

21. Yang, S. et al. Spatiotemporal dynamics of hemorrhagic fever with renal syndrome in Jiangxi province, China. entific Reports.

22. Yue, Y. et al. Spatio-temporal patterns of scrub typhus in mainland China, 2006-2017. PLoS neglected tropical diseases 13, e0007916, doi:10.1371/journal.pntd.0007916 (2019).

23. Gao, Y. et al. Epidemiological Characteristics and Spatiotemporal Patterns of Typhus Group Rickettsiosis at the County Level in China, 2005-2017. International journal of infectious diseases: IJID : official publication of the International Society for Infectious Diseases, doi:10.1016/j.jijid.2019.11.018 (2019).

24. Elliott, P. \& Wartenberg, D. Spatial Epidemiology: Current Approaches and Future Challenges. Environmental Health Perspectives 112, 998-1006 (2004).

25. Jiangxi Statistical Yearbook 2018. http://www.jxstj.gov.cn/resource/nj/2018CD/indexeh.htm. Statistic Bureau of Jiangxi. Accessed 10 Nov 2020.

26. Wang, J. B. \& Song, J. Y. Distribution and geographical regionalization of Chigger mites in Jiangxi province. Jiangxi Plant Protection 17, 1-5 (1994).

27. Kulldorff, M. A spatial scan statistic. Communications in Statistics 26, 1481-1496 (1997).

28. Wu, Y. C. et al. Spatiotemporal Dynamics of Scrub Typhus Transmission in Mainland China, 20062014. PLoS neglected tropical diseases 10, e0004875, doi:10.1371/journal.pntd.0004875 (2016).

29. Wei, Y. et al. Rapid increase of scrub typhus: an epidemiology and spatial-temporal cluster analysis in Guangzhou City, Southern China, 2006-2012. PloS one 9, e101976, doi:10.1371/journal.pone.0101976 (2014).

30. Zheng, L. et al. Epidemic characteristics and spatio-temporal patterns of scrub typhus during 20062013 in Tai'an, Northern China. Epidemiology and infection 143, 2451-2458, doi:10.1017/s0950268814003598 (2015).

31. Yu, H. et al. Scrub typhus in Jiangsu Province, China: epidemiologic features and spatial risk analysis. BMC infectious diseases 18, 372, doi:10.1186/s12879-018-3271-x (2018).

32. Chen, R. et al. Analysis of epidemiological characteristics of four natural-focal diseases in Shandong Province, China in 2009-2017: A descriptive analysis. PloS one 14, e0221677, doi:10.1371/journal.pone.0221677 (2019).

33. Paris, D. H., Shelite, T. R., Day, N. P. \& Walker, D. H. Unresolved problems related to scrub typhus: a seriously neglected life-threatening disease. The American journal of tropical medicine and hygiene 89, 301-307, doi:10.4269/ajtmh.13-0064 (2013). 
34. Kelly, D. J., Foley, D. H. \& Richards, A. L. A Spatiotemporal Database to Track Human Scrub Typhus Using the VectorMap Application. PLoS neglected tropical diseases 9, e0004161, doi:10.1371/journal.pntd.0004161 (2015).

35. Walker, D. H. Scrub Typhus - Scientific Neglect, Ever-Widening Impact. N Eng/ J Med 375, 913-915, doi:10.1056/NEJMp1608499 (2016).

36. Yao, H. et al. The scrub typhus in mainland China: spatiotemporal expansion and risk prediction underpinned by complex factors. Emerging microbes \& infections 8, 909-919, doi:10.1080/22221751.2019.1631719 (2019).

37. Wei, Y. et al. Climate variability, animal reservoir and transmission of scrub typhus in Southern China. PLoS neglected tropical diseases 11, e0005447, doi:10.1371/journal.pntd.0005447 (2017).

38. Kuo, C. C. et al. Cascading effect of economic globalization on human risks of scrub typhus and tickborne rickettsial diseases. Ecol App/22, 1803-1816, doi:10.1890/12-0031.1 (2012).

39. Zhi-Yang, S. et al. Analysis on the epidemiological characteristics of scrub typhus in Fuzhou from 2011 to 2015. Chinese Journal of Disease Control \& Prevention (2018).

40. Yong, L., Ren-Fa, H. \& Xiao-Jun, H. U. Risk factors of scrub typhus in Ganzhou city of Jiangxi province: a case control study. Chinese Journal of Public Health (2018).

41. Wang, X. Z. \& Liu, S. B. Analysis on Rural Surplus Labor in Jiangxi Province. East China Economic Management 23, 23-26 (2009).

42. Rural Labor Migration and Householdsī1/4\ Land Rental Behavior: Evidence from China. China and World Economy (2018).

43. Watt, G. \& Parola, P. Scrub typhus and tropical rickettsioses. Curr.opin.infect.dis 16, 429-436 (2003).

\section{Figures}




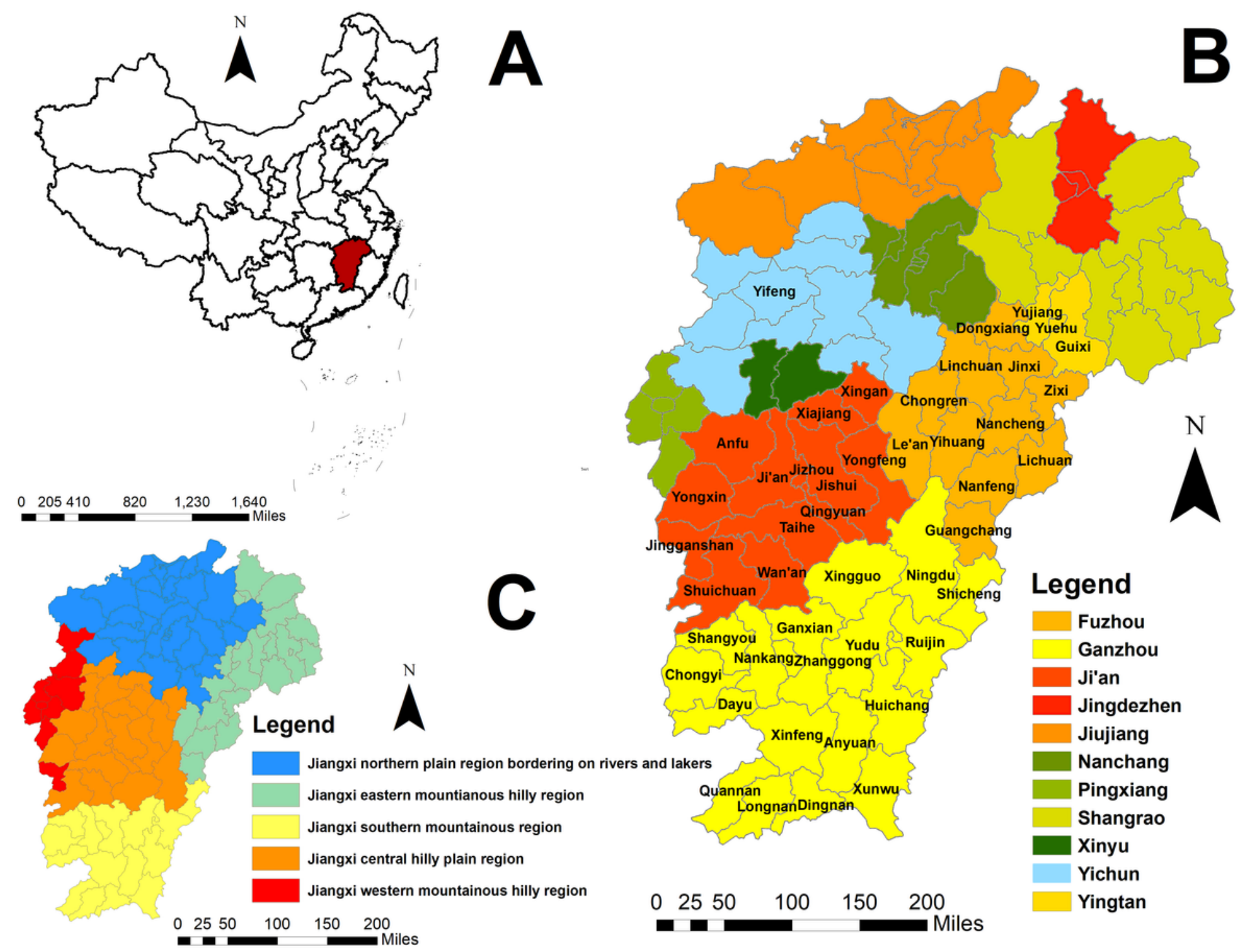

Figure 1

The location of the study area. (A) Location of Jiangxi province, in China. (B) Administrative division of the study area. (C) The geographic distribution of five zoographic regions. (Version 10.4 ESRI, Redlands, CA, USA, https ://www.esri.com/softw are/arcgi s/arcgi s-for-desktop). Note: The designations employed and the presentation of the material on this map do not imply the expression of any opinion whatsoever on the part of Research Square concerning the legal status of any country, territory, city or area or of its authorities, or concerning the delimitation of its frontiers or boundaries. This map has been provided by the authors. 


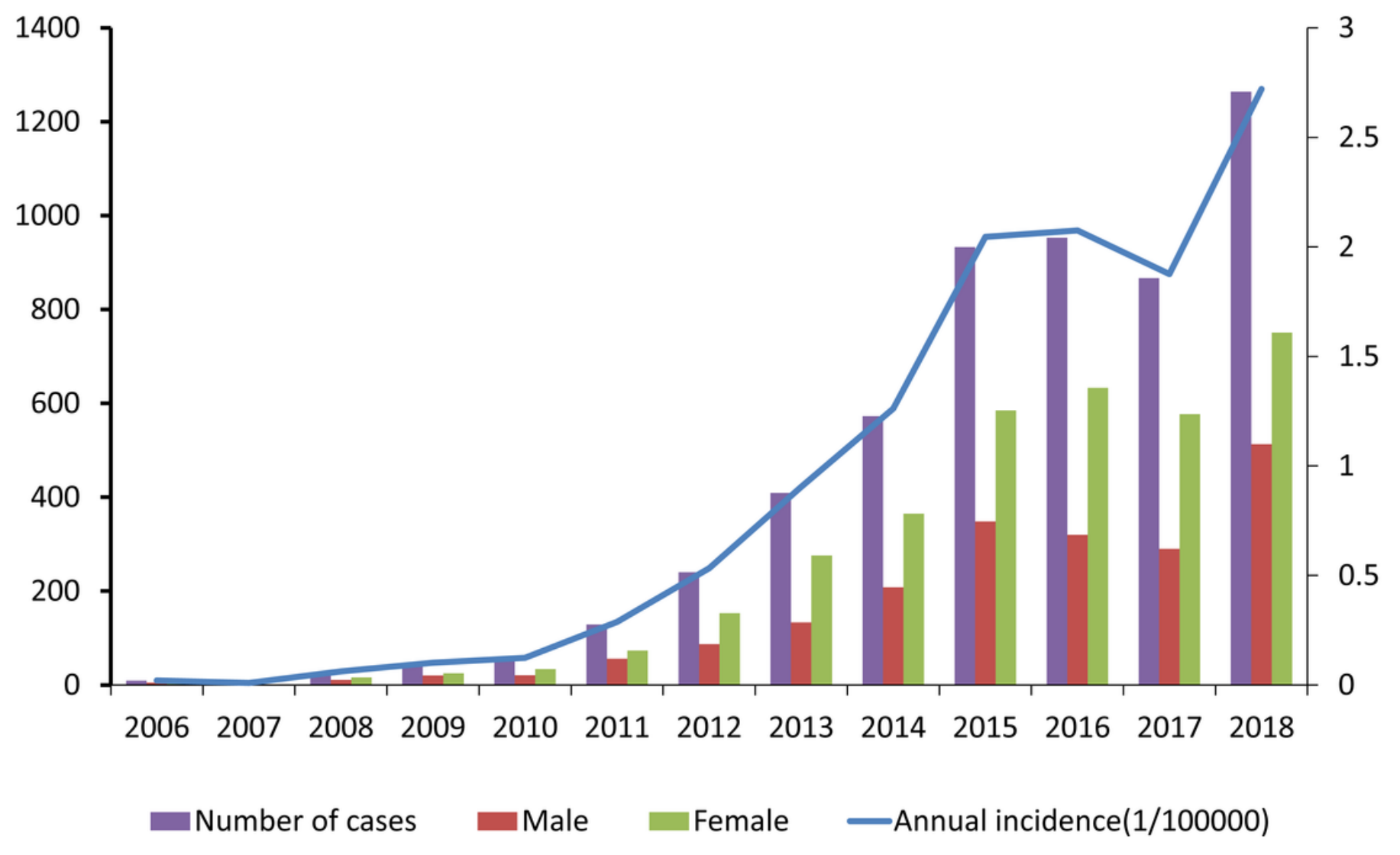

Figure 2

Scrub typhus case numbers and trends in Jiangxi from 2006 to 2018. 


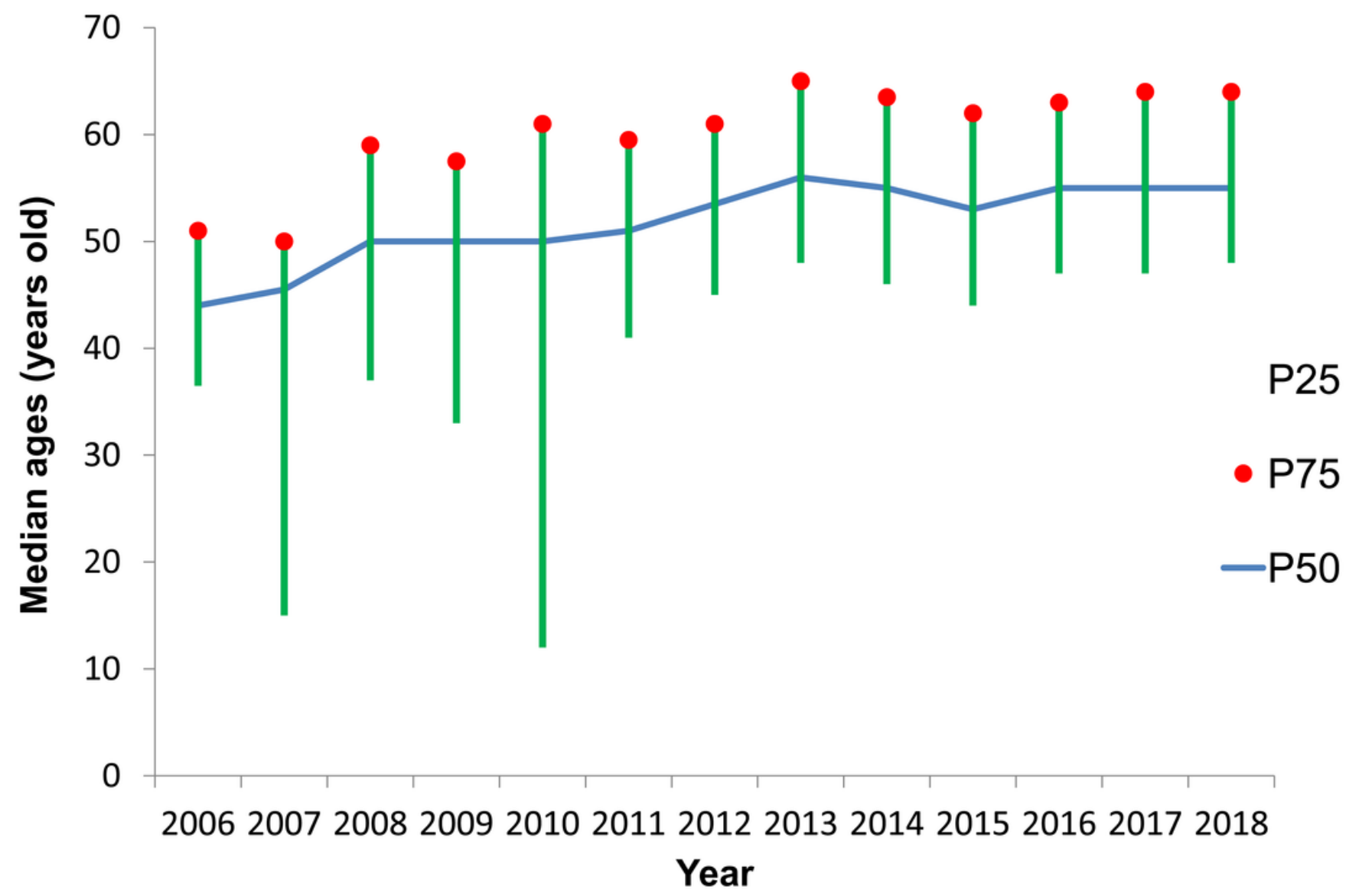

Figure 3

Median ages of the typhus group rickettsiosis cases in Jiangxi from 2006 to 2018. 


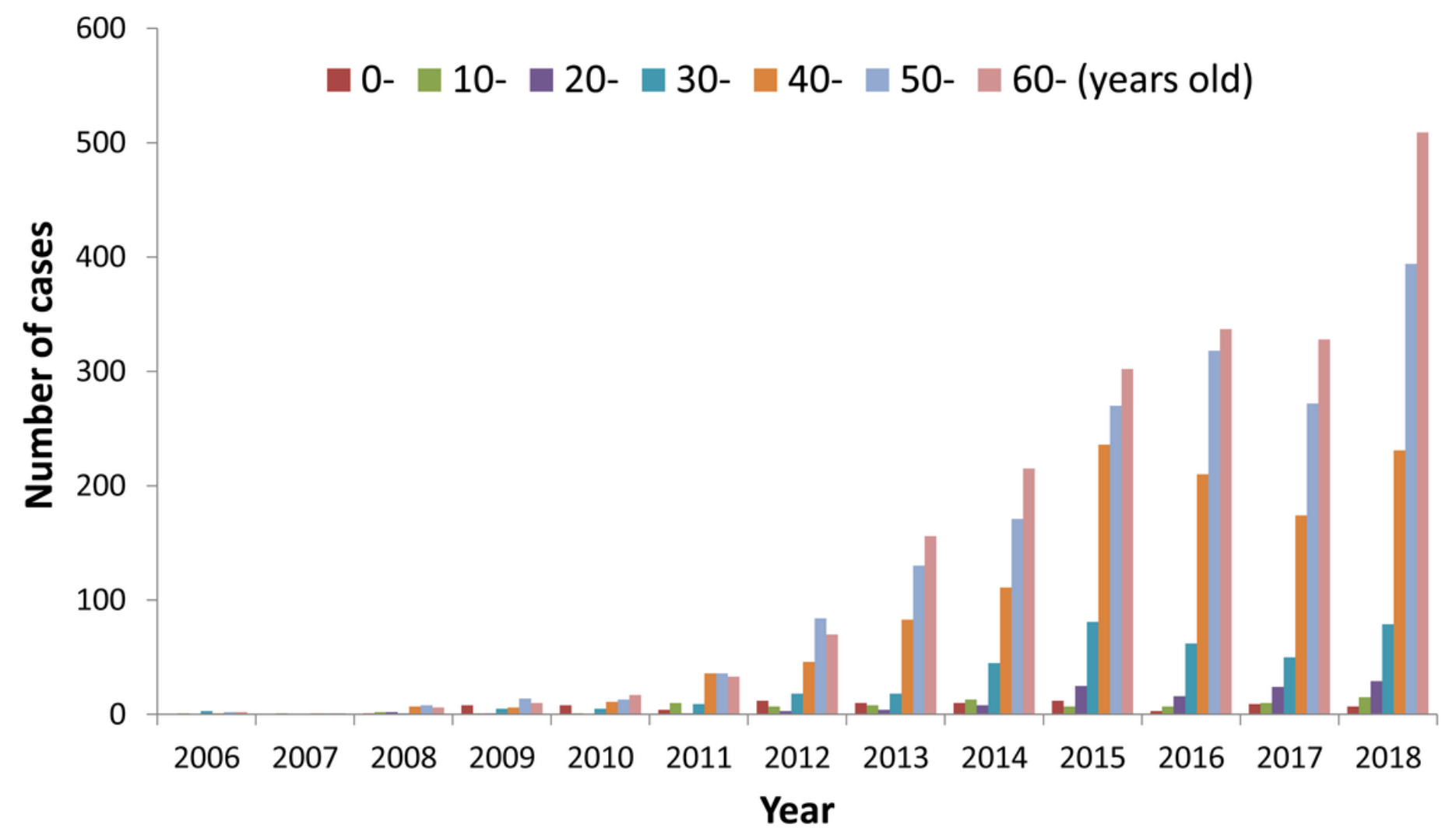

Figure 4

Age distribution of the scrub typhus cases in Jiangxi from 2006 to 2018. 


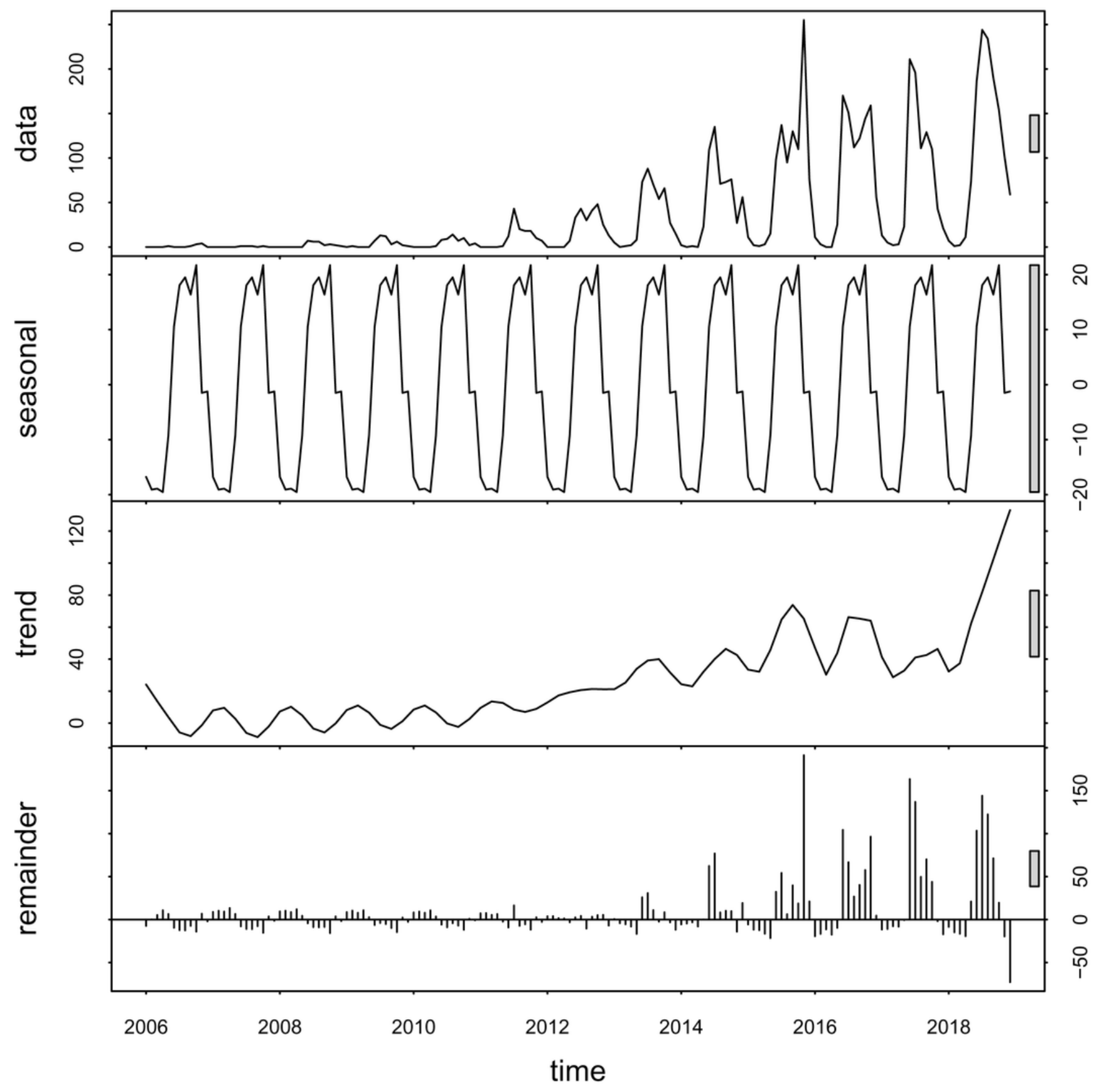

Figure 5

Decomposed scrub typhus cases in the study area from 2006 to 2018. These maps were generated by ArcGIS software (Version 10.4 ESRI, Redlands, CA, USA, https ://www.esri.com/software/arcgis/arcgisfor-deskt op). 


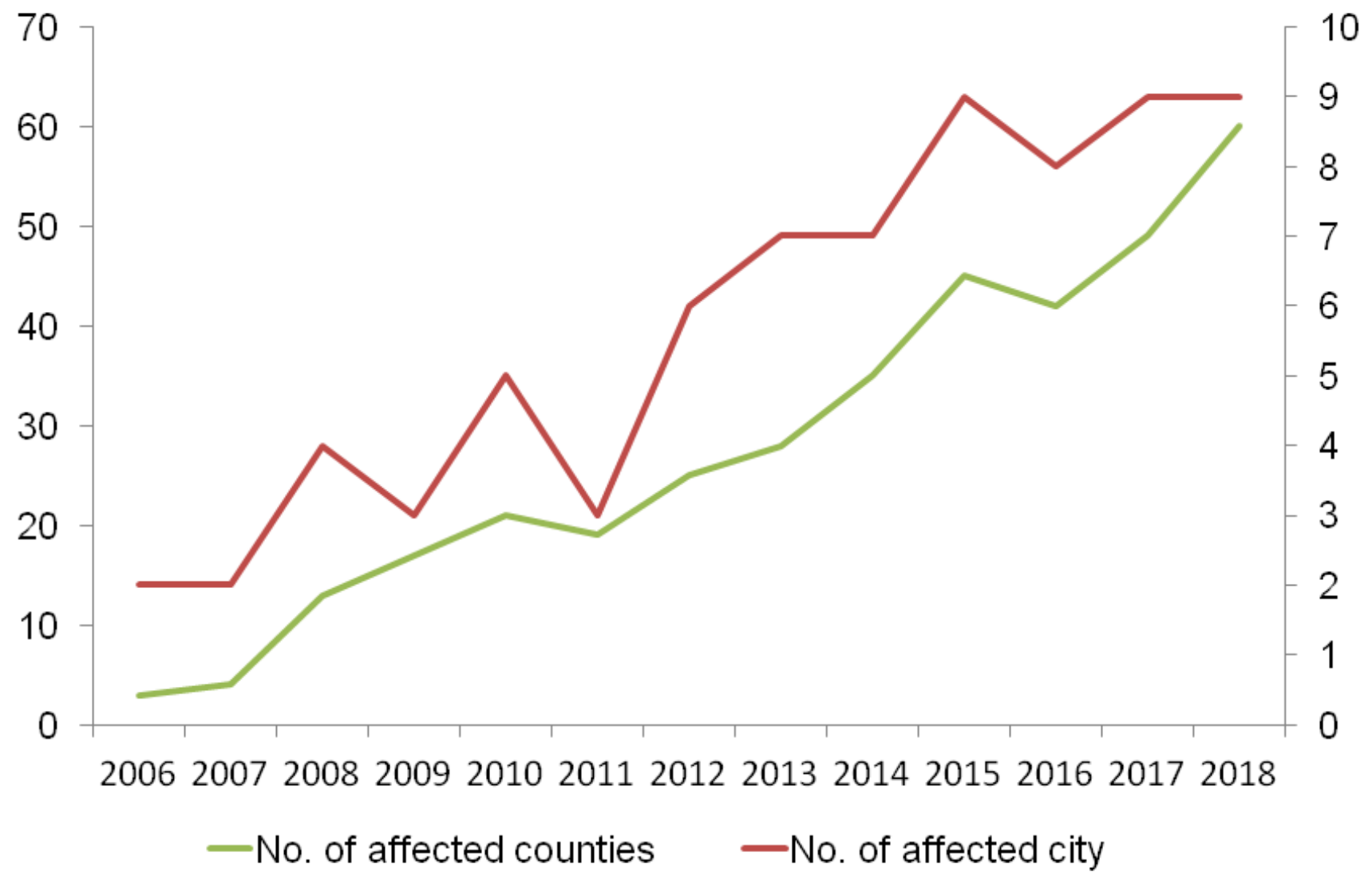

Figure 6

Numbers of areas with scrub typhus cases in Jiangxi from 2006 to 2018. 


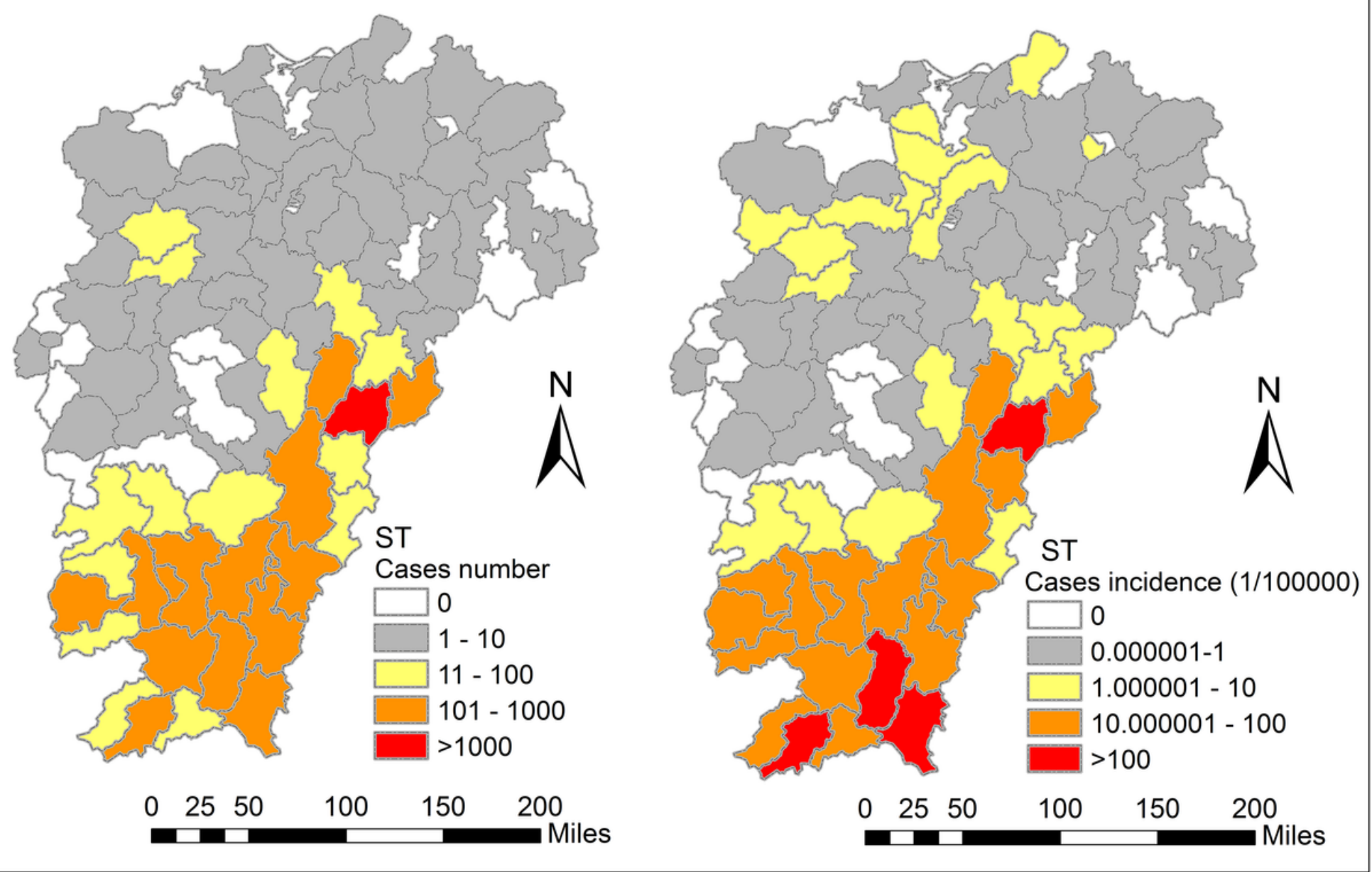

Figure 7

Spatial distribution of scrub typhus cases in Jiangxi from 2006 to 2018. (A) Spatial distribution of ST cases in Jiangxi from 2006 to 2018. (B) Cumulative incidence of the ST cases in Jiangxi from 2006 to 2018. These maps were generated by ArcGIS software (Version 10.4 ESRI, Redlands, CA, USA, https ://www.esri.com/software/arcgis/arcgis-for-deskt op). Note: The designations employed and the presentation of the material on this map do not imply the expression of any opinion whatsoever on the part of Research Square concerning the legal status of any country, territory, city or area or of its authorities, or concerning the delimitation of its frontiers or boundaries. This map has been provided by the authors. 

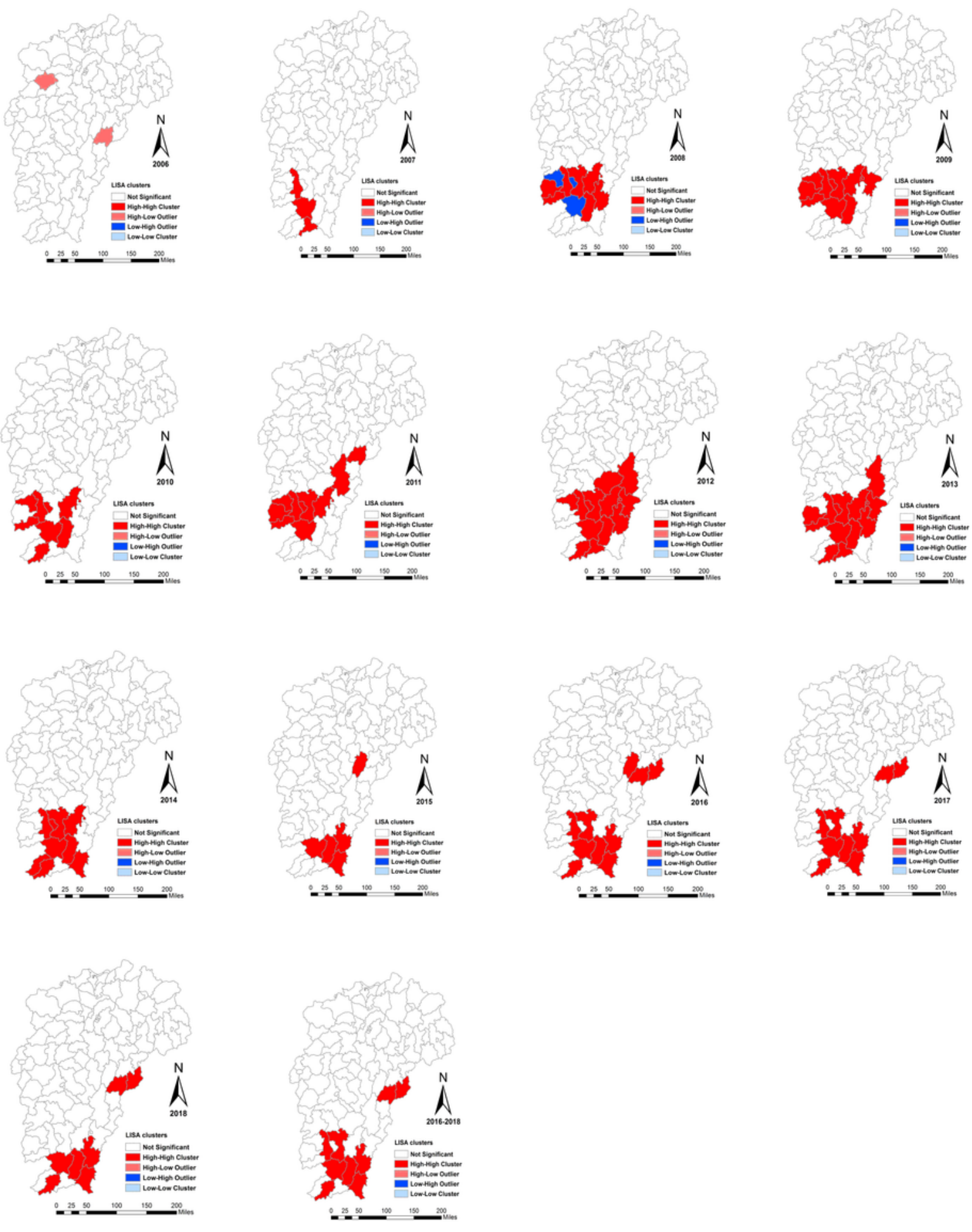

\section{Figure 8}

Local indicators of spatial association cluster maps for scrub typhus in the study area from 2006 to 2018. These maps were generated by ArcGIS software (Version 10.4 ESRI, Redlands, CA, USA, https ://www.esri.com/software/arcgis/arcgis-for-deskt op). Note: The designations employed and the presentation of the material on this map do not imply the expression of any opinion whatsoever on the part of Research Square concerning the legal status of any country, territory, city or area or of its 
authorities, or concerning the delimitation of its frontiers or boundaries. This map has been provided by the authors.
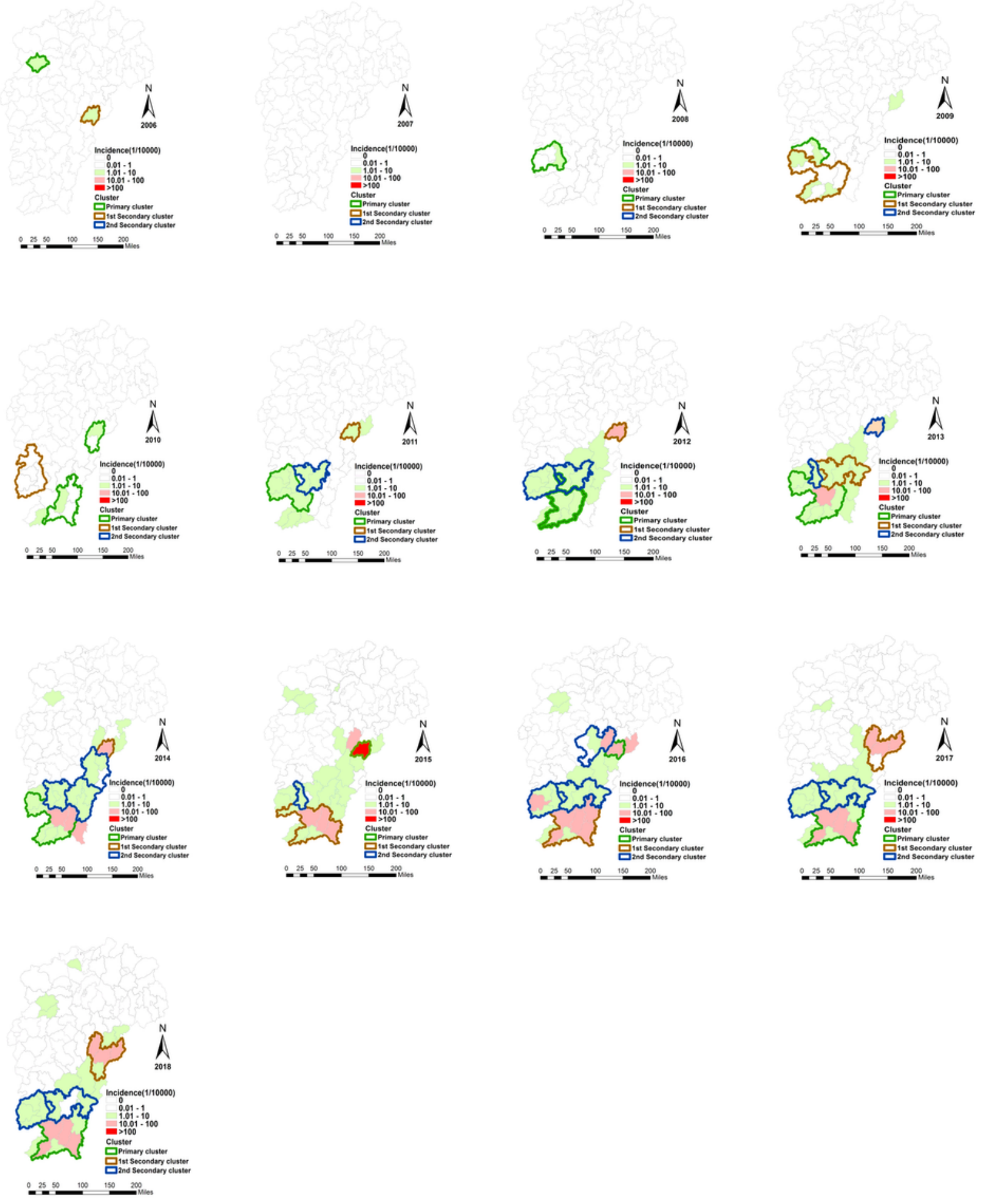

\section{Figure 9}

Yearly spatiotemporal clusters overlay with an annual incidence of scrub typhus in Jiangxi from 2006 to 2018. Yearly spatiotemporal clusters were detected using a circular scan window with the maximum spatial size of $5 \%$ of the population at risk and a maximum temporal size of $10 \%$ of the study period. 
These maps were generated by ArcGIS software (Version 10.4 ESRI, Redlands, CA, USA, https ://www.esri.com/software/arcgis/arcgis-for-deskt op). Note: The designations employed and the presentation of the material on this map do not imply the expression of any opinion whatsoever on the part of Research Square concerning the legal status of any country, territory, city or area or of its authorities, or concerning the delimitation of its frontiers or boundaries. This map has been provided by the authors.

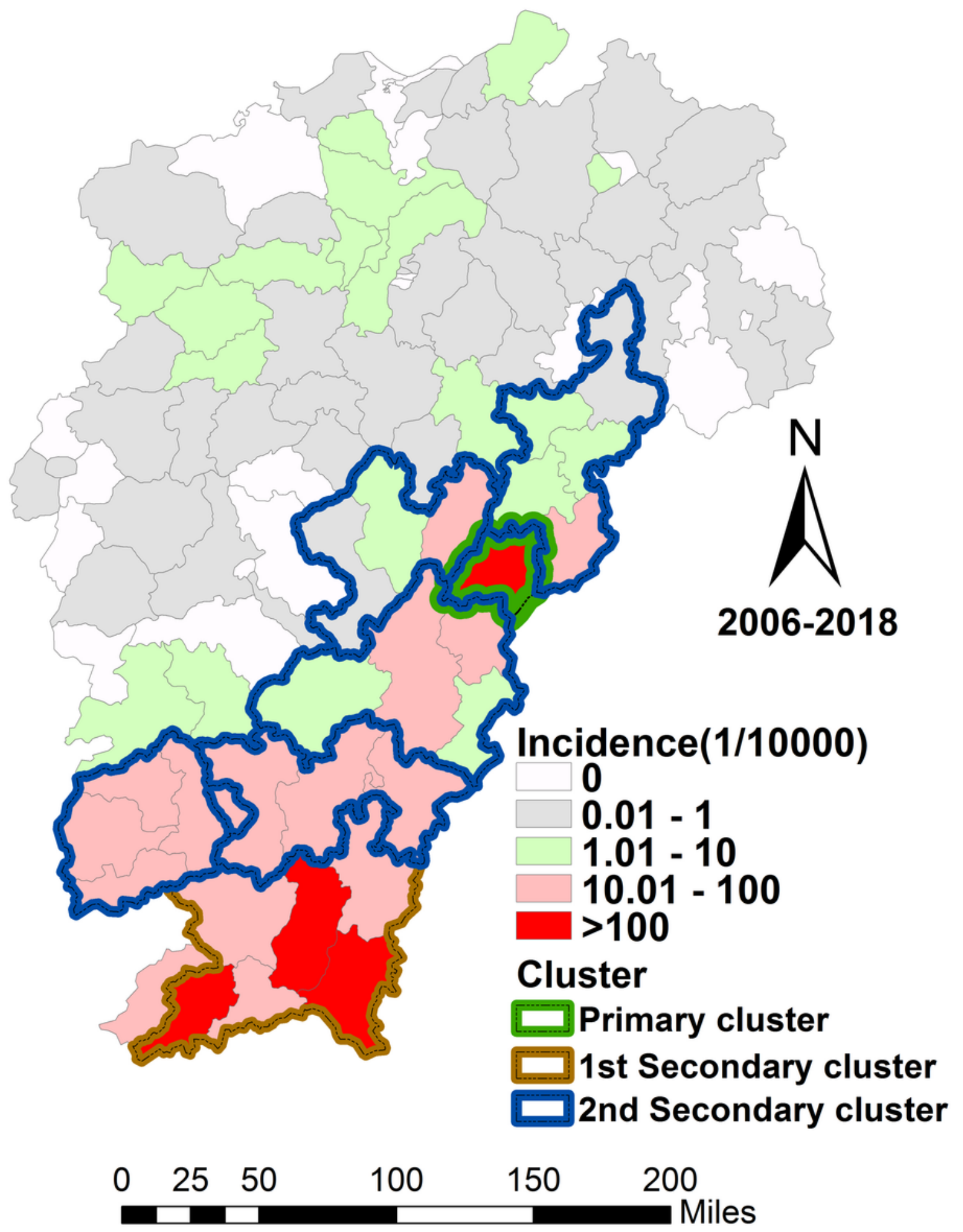

Figure 10 
Spatiotemporal clusters overlay with the annual average incidence of scrub typhus across the period of 2006-2018 in the study area. These maps were generated by ArcGIS software (Version 10.4 ESRI, Redlands, CA, USA, https ://www.esri.com/software/arcgis/arcgis-for-deskt op). Note: The designations employed and the presentation of the material on this map do not imply the expression of any opinion whatsoever on the part of Research Square concerning the legal status of any country, territory, city or area or of its authorities, or concerning the delimitation of its frontiers or boundaries. This map has been provided by the authors.

\section{Supplementary Files}

This is a list of supplementary files associated with this preprint. Click to download.

- SupplementaryMaterial.rar 